\title{
Contribution of Interventional Radiology to the Management of COVID-19 patient
}

\author{
Lorenzo Monfardini $^{1} \cdot$ Claudio Sallemi $^{1} \cdot$ Nicolò Gennaro $^{2} \cdot$ Vittorio Pedicini $^{3}$ • \\ Claudio Bnà ${ }^{1}$
}

Received: 14 March 2020/Accepted: 26 March 2020/Published online: 22 April 2020

(C) Springer Science+Business Media, LLC, part of Springer Nature and the Cardiovascular and Interventional Radiological Society of Europe (CIRSE) 2020

To the Editor,

The COVID-19 outbreak is currently spreading throughout northern Italy and is forcing a rapid change of clinical priorities and organizational logistics. In this dramatic scenario, angio-suites and operatory rooms only admit urgent procedures and the interventional radiology (IR) service is mostly dedicated to assisting the emergency department in dealing with SARS-CoV-2-positive patients. We share a case of pleural drainage in one COVID-19 patient and provide the IR community with some practical recommendations regarding the management of this particular category of patients.

A 63-year-old woman with metastatic ovarian cancer and recurrent malignant pleural effusion came to our institutional ER with fever $\left(39^{\circ} \mathrm{C}\right)$ and dyspnea at rest. Being our institute located in a severely involved area of the COVID19 outbreak, clinical symptoms were compatible with COVID-19 infection. Laboratory parameters included hemoglobin 10.7, white blood cells 3.98, C-reactive protein 43.5, arterial $\mathrm{pH} 7.5, \mathrm{pO}_{2} 53 \mathrm{mmHg}, \mathrm{pCO}_{2} 31 \mathrm{mmHg}$ and blood lactate concentration $1 \mathrm{mmol} / \mathrm{L}$. Although the patient underwent a right pleurodesis 6 months ago, chest X-ray showed bilateral pleural effusion (loculated on the right side) as well as interstitial thickening (Fig. 1A), a common

Nicolò Gennaro

nicolo.gennaro@st.hunimed.eu

1 Department of Radiology, Fondazione Poliambulanza Istituto Ospedaliero, Brescia, Italy

2 Radiology Residency Program, Humanitas University, Via R.L. Montalcini 4, 20090 Pieve Emanuele, Italy

3 Department of Radiology, Humanitas Clinical and Research Hospital, Rozzano, Italy imaging feature in COVID-19. She underwent a naso-/ oropharyngeal swab which turned positive for SARS-CoV2 nucleic acid after being processed through a real-time reverse transcriptase polymerase chain reaction (Novel Coronavirus PCR Fluorescence Diagnostic Kit, BioGerm Medical Biotechnology). She started as an inpatient with 1 week of non-invasive assisted ventilation that reduced fever, clinical symptoms and normalized inflammatory laboratory values such as C-reactive protein. Still, respiratory function and blood oxygen levels remained poor despite an increase in $\mathrm{FiO}_{2}$ from 60 to $70 \%$. Consequently, percutaneous drainage was requested with the aim of improving ventilation and blood oxygen saturation. The procedure was performed at the bedside in an isolated ward dedicated to COVID-19 patients. The operator wore an FFp2 mask, double gloves, protective glasses and a surgical gown (Fig. 2). After local anesthesia, two 8F multi-hole drainages were inserted bilaterally using dedicated US equipment in the isolated ward as image guidance. The procedure lasted approximately $1 \mathrm{~h}$ and was well tolerated. Around $1800 \mathrm{~mL}$ was drained in the next $5 \mathrm{~h}$ from both sides. All disposable equipment and devices were then immediately discarded in specific containers. Post-procedural chest X-rays showed a marked increase in the ventilated parenchyma. Along with clinical symptoms, also laboratory markers rapidly improved and blood saturation levels reached the normal range $\left(\mathrm{SpO}_{2} 98 \%\right)$. The patient switched from assisted ventilation to a simple face mask (2 $\mathrm{L} / \mathrm{min} \mathrm{O}_{2}$ ) and maintained normal peripheral capillary oxygen saturation. At the time of writing, she is alive and close to hospital discharge.

The current COVID-19 outbreak is causing a massive overcrowding of admitted patients for symptomatic pneumonia. Patient presentation ranges from mild symptoms to 


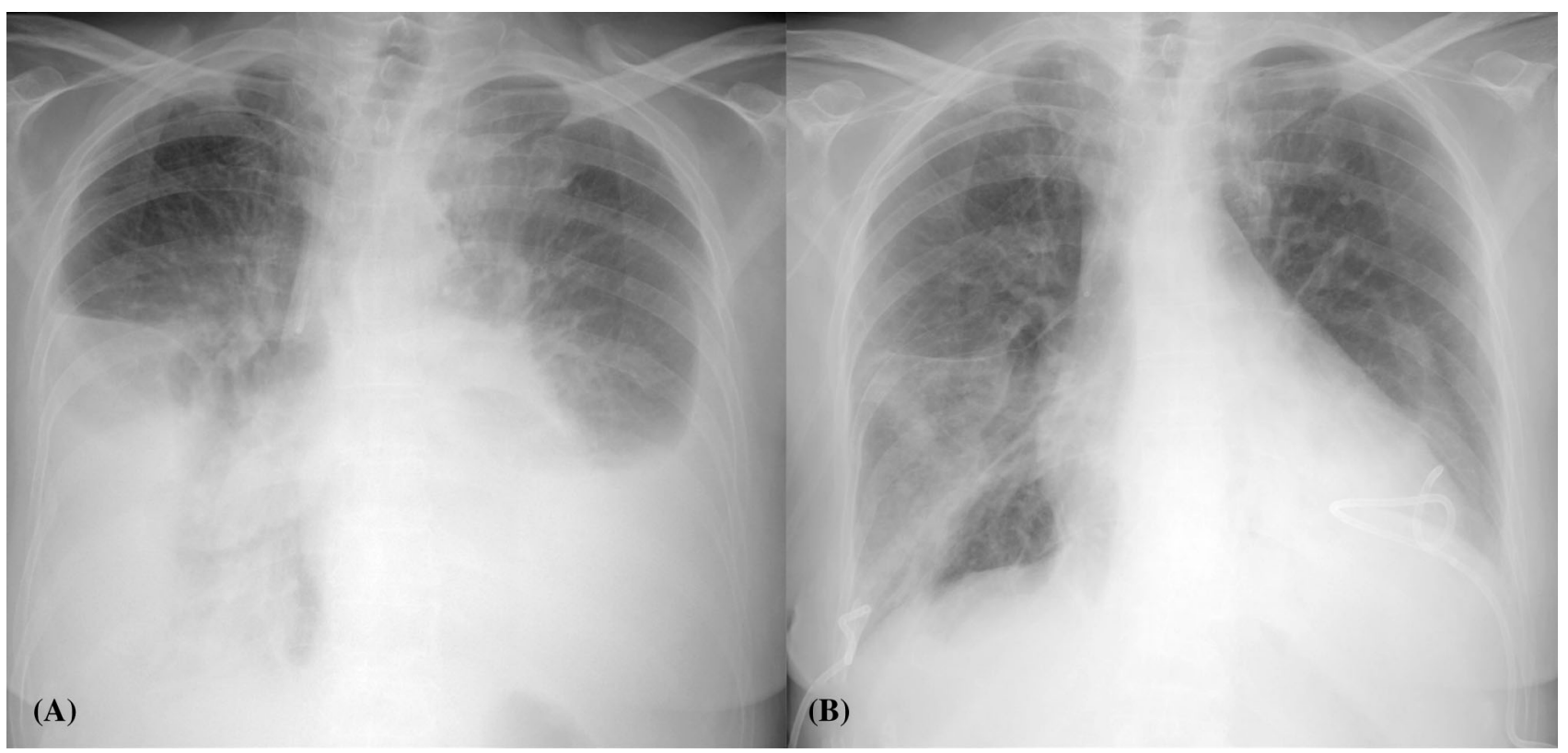

Fig. 1 A Chest X-ray shows bilateral pleural effusion and diffuse interstitial thickening. B Chest X-ray performed $24 \mathrm{~h}$ after bilateral pleural drainages shows nearly complete resolution of the pleural effusions on both sides

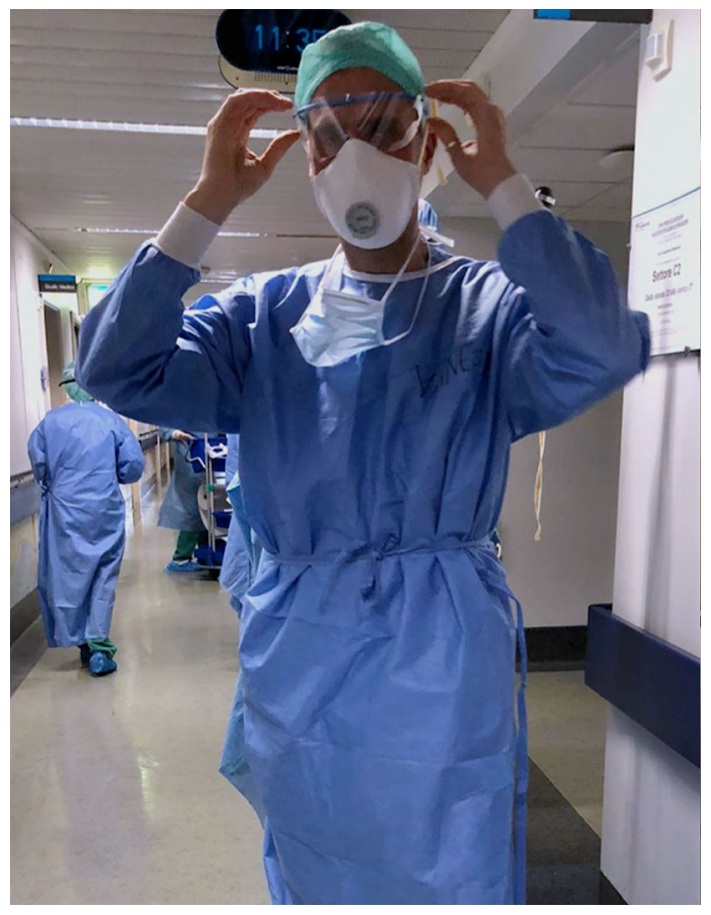

Fig. 2 Operator wearing protective equipment before the procedure

severe respiratory syndrome due to extended parenchymal involvement, especially in the advanced-phase disease (8-14 days after the onset of first symptoms) [1].

Pleural effusion is not typical of COVID-19 infection, but is a common finding in metastatic oncologic patients and may severely impact respiratory function. Whether the infection concurred to the recurrence of the pleural effusion is not known, but pleural effusion in COVID-19 patients has been described as a poor prognosticator of clinical evolution [2]. Being ventilation the first line treatment for COVID-19 patients, pleural drainage is recommended to improve oxygenation.

US-guided thoracic interventions like pleural drainage is a safe and practical procedure that can be performed at the bedside with due arrangements for operator's safety in case of infectious patients [3]. The versatility of US-guided procedures fits well with the need to entail procedures on isolated patients in separate places and times [4]. In fact, the possibility to perform the procedure at the bedside avoids the spread of disease outside the dedicated COVID19 ward and does not require discontinuation of assisted ventilation.

This is not the first time that interventional radiologists have been called to actively contribute to an infectious outbreak. In 2003, the SARS outbreak raised awareness among the IR community about the role of our specialty in such a delicate scenario. Lau et al. [5] proposed decentralization of the service, predilection of US-guided procedures, rigorous triage and strict respect of safety measures as key elements for delivering high-quality IR care.

We acknowledge that in this historic moment the overcrowding of the emergency units may hinder multidisciplinary collaboration. However, considering that 
COVID-19 is a rapidly changing respiratory syndrome with unpredictable clinical evolution, a smooth collaboration between IR and all the health workers involved in the management of COVID-19 patients will be critical in the upcoming months to effectively distribute the workload and timely ensure the best care possible.

In conclusion, IR units should be responsive and equipped to deal with COVID-19 outbreak, as they may play a key role in the optimization of patient ventilation through minimally invasive procedures.

Authors Contributions All the authors mentioned in the manuscript have made a significant contribution to this manuscript, agreed for authorship, read and approved the manuscript and agreed to its submission to CVIR.

Funding The authors state that this work has not received any funding.

\section{Compliance with Ethical Standards}

Conflict of interest The authors declare that they have neither financial nor non-financial conflicts of interest.

Ethical Approval and Informed Consent Both Institutional Review Board and Informed consent were obtained.

Consent for Publication Consent for publication was obtained for every individual person's data included in the study.
Statistics and Biometry No complex statistical methods were necessary for this paper.

Study Subjects or Cohorts Overlap Study subjects or cohorts have not been previously reported.

\section{References}

1. Zhang J-J, Dong X, Cao Y-Y, et al. Clinical characteristics of 140 patients infected by SARS-CoV-2 in Wuhan, China. Allergy. 2020. https://doi.org/10.1111/all.14238.

2. Zhou S, Wang Y, Zhu T, Xia L. CT features of coronavirus disease 2019 (COVID-19) Pneumonia in 62 patients in Wuhan, China. AJR Am J Roentgenol. 2020. https://doi.org/10.2214/AJR.20. 22975.

3. Vetrugno L, Guadagnin GM, Orso D, et al. An easier and safe affair, pleural drainage with ultrasound in critical patient: a technical note. Crit Ultrasound J. 2018;10:18. https://doi.org/10. 1186/s13089-018-0098-z.

4. Da Zhuang K, Tan BS, Tan BH, et al. Old threat, new enemy: is your interventional radiology service ready for the coronavirus disease 2019? Cardiovasc Intervent Radiol. 2020. https://doi.org/ 10.1007/s00270-020-02440-6.

5. Lau T-N, Teo N, Tay K-H, et al. Is your interventional radiology service ready for SARS?: the Singapore experience. Cardiovasc Intervent Radiol. 2003;26:421-7. https://doi.org/10.1007/s00270003-0143-5.

Publisher's Note Springer Nature remains neutral with regard to jurisdictional claims in published maps and institutional affiliations. 